\title{
Teaching Software Based on WeChat Platform
}

\author{
Qiufeng Shang \\ Department of Electronics and Communication \\ North China Electric Power University \\ Baoding, China
}

\author{
Wenjie Qin \\ Department of Electronics and Communication \\ North China Electric Power University \\ Baoding, China
}

\begin{abstract}
The higher education in China has attracted more and more attention to the cultivation of engineering innovation ability of college students. Based on WeChat Platform, the teaching software has been developed and can provide functions such as data sharing, remote program debugging, mobile phone network login, experience sharing and discussion, sharing labs, online teaching, etc. With advantages of high-efficiency share, this software not only improves the utilization rate of professional laboratories in colleges and universities, but also enhances the teaching effect. By means of this software it is conducive to the upgrading of experimental equipment and improve students' innovative design capabilities.
\end{abstract} ability

Keywords-teaching software; WeChat; sharing; innovation

\section{INTRODUCTION}

How to achieve the organic integration of the theory courses teaching and practice project to enhance the teaching effect? And how to combine the utilization of professional laboratories with improving the quality of innovative entrepreneurial activities? In order to solve these problems, the teaching software based on framework of WeChat public platform has been developed in this paper.

Taking into account the characteristics of mobile terminals, design work includes two parts: the front end and the back end. The front end mainly refers to the application of HTML, CSS, JavaScript technologies to implement webpage jump. In order to integrate with the background development of the platform, the list page template and split template were implemented. WeChat version compatibility was debugged. SAE Sina cloud server was used as the back-end server. The back end mainly implements the logical function of the webpage, returns the user URL response, uses the database to realize the registration of the user's information, and performs real-time management recording of the sharing request of the device resource, using the PHP language in the webpage framework to realize discussion and information exchange among users. [1-10]

\section{FUNCTIONS AND FEATURES OF THE TEACHING SOFTWARE}

As shown as Fig.1, the WeChat platform software includes three first-level menus: user center, common services and interactive assistance featured functions. There are multiple secondary menus: user registration, user login, personal information, device reservation, device upload, news

This work is supported by (13001802) the research project on the reform of higher education in Hebei, China. information, video Q\&A, data download, question sections and interactive assistance. The main interface of the teaching software is shown as Fig.2.

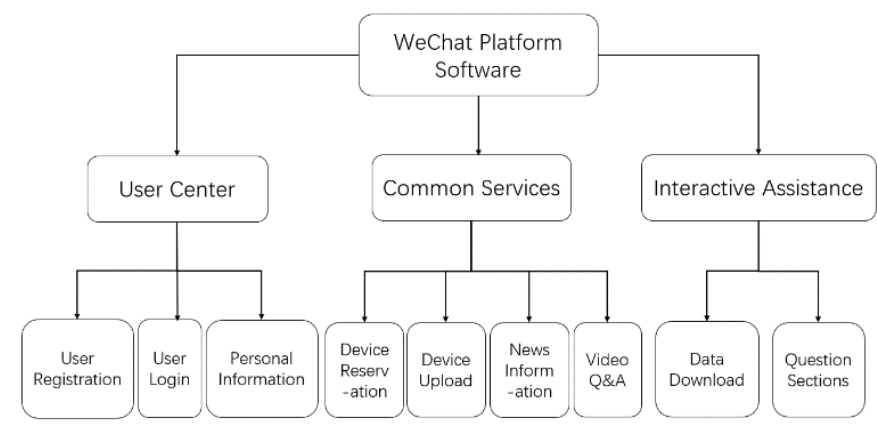

Fig. 1. The WeChat platform software.

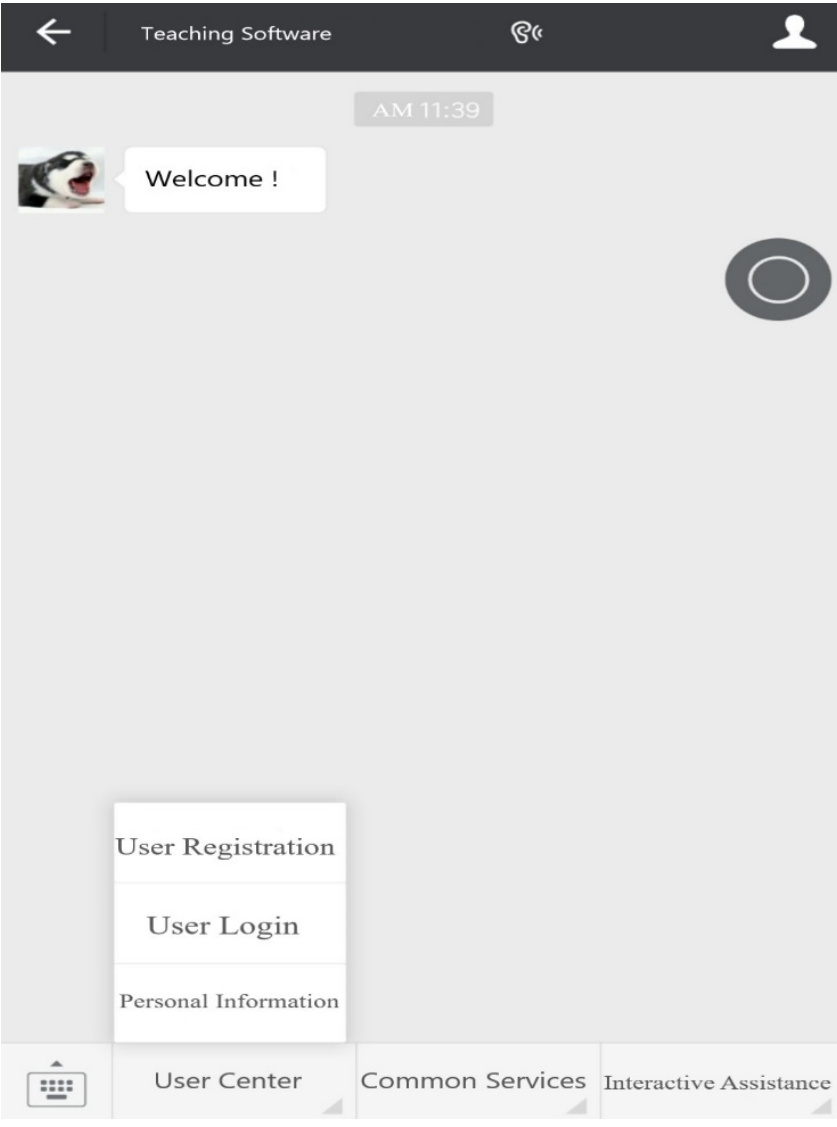

Fig. 2. The main interface of the teaching Software. 


\section{A. Functions of the Teaching Software}

\section{1) User center}

This module mainly implements user information management, including user registration and user login. The registration page refreshes the basic information of the submitted user, and the administrator performs regular updates of the database.

In order to realize the micro-platform construction with the core concept of interactive sharing and collaborative development, the user system supplies interaction among users. Users can use more rich features only after logging in, and the implementation of featured function requires users to provide their own information. These two parts depend on each other and promote each other.

\section{2) Common services}

This module is mainly based on resource sharing. Function menus are designed such as device upload and device reservation . Through device upload, users can register their own idle development equipment in this WeChat platform system, so that other users in need can register and borrow from this micro platform. In this way, the development and learning resources of the school can be shared with other users of the platform. At the same time, the idle equipment of the school laboratory will also be registered on the platform, and the utilization efficiency of the teaching resources will also be improved. The equipment reservation menu is mainly to realize the registration of information with the reservation person, so that the micro-platform can better serve the reservation process.

Typical services including:

a) Equipment Reservation: The administrators write the information submitted by users to the database. By comparison with the audit, the user is given an appointment reply when the request is met. When the user need to use certain device, they can submit applications through the platform. The background programme will filter the database based on the submitted device name and type, and help users to make an appointment directly.

b) Resource Share: The user can use this function to register all hardware devices, as well as related product manuals, functional instructions, example programs, and so on.

c) Video Course: The platform provides a video list. In view of the problems that often happen in the practical process, the video explanation is carried out, and the user can watch it online.

d) File Download: The platform provides a keyword response function, for example, the user sends "data" to the platform, it can directly return the data download link.

\section{3) Interactive assistance module}

This module is mainly based on the core concept of collaborative development. A community to publish problems are designed. In the process of development and learning, there are often many problems that cannot be expressed easily by words. In response to this situation, a community where issues can be published is designed. Every user of the microplatform can see the questions posted and answer them. The solution is mainly divided into remote assistance and video answering. Because WeChat does not provide remote assistance interface, the micro-platform transfers this problem to the computer for remote assistance.

Typical services including:

a) Question Forum: The user can display their questions on the platform. The others can communicate and reply online.

b) Request Assistance: When users need to operate on the experimental equipment remotely, the background programmers help to connect the messages.

In addition to the main functional modules described above, the maintenance module is also needed.

For example:

a) Platform Optimization: The developers need to optimi-ze browser interface, repair vulnerabilities and improve the operation performance.

b) Browser Adaptation: When WeChat software version is updated, the developers need to test and adjust the rendering results of browser kernels in time, so as not to affect interface layout.

\section{B. Features of the Teaching Software}

The practical teaching WeChat platform constructed in this paper supports mobile terminal devices. Different from the traditional network teaching platform that can only be accessed through PC-side browser using B/S architecture, users can conveniently access the teaching software through mobile phone WeChat anytime and anywhere to obtain services. The platform enables users to realize collaborative development through the video service provided by WeChat and remote assistance more conveniently and effectively. Compared with the common WeChat public platform, the platform is more focused on teaching interaction and development collaboration, rather than simply pushing messages to users. Compared with the traditional network teaching platform ,users can get interactive and remote control experience.

The teaching platform constructed in this paper can support the teaching and training activities inside and outside the class. This platform is different from the general WeChat public number with browsing function, and avoids the problems of insufficient dynamic interaction ability and generalization of the general network teaching platform $[4,5]$. The main features of the platform is sharing and remote control.

\section{SHARING FUNCTION}

The sharing in the software includes the sharing of materials and equipment. Data can be uploaded and downloaded, including categories such as video, audio, pictures and text. The device control can be transferred. The user remotely control and debug the shared equipment, saving teaching resources. The shared equipment may come from laboratories, teachers and students, or from engineer such as alumni and teaching equipment manufacturers. The equipment of enthusiastic alumni and teaching equipment manufacturers is often more advanced than the equipment of the school 
laboratory. Sharing can improve the engineering ability of students and teachers.

With the rapid development of information technology, the update speed of the practice teaching platform for information courses is getting faster and faster. If large-scale short-cycle elimination of teaching equipment in professional laboratories is inevitably subject to financial pressure, and the construction cycle is difficult to guarantee. Therefore, it is possible to purchase a few of the improved equipment in stages on the basis of the existing equipment. At the same time, in order to meet the needs under limited venues and a limited number of experimental equipment, this paper proposes to explore a shared practice teaching mode, so that students can use the sharing mechanism to implement online and offline learning through the practical teaching WeChat platform and remotely complete part of the program debugging, which can improve the utilization of experimental equipment and achieve better teaching results.

Sharing function can accelerate the learning process of students and improve the learning effect. For example, hardware knowledge is a difficult point in professional learning. If every student is working on it, personal ability and teaching expenses cannot be guaranteed. Using the production and debugging videos of previous students can intuitively learn the online learning process. Through the online Q\&A and discussion of the platform, the hardware design ability of the students can be improved overall.

Part of the shared function display interface is shown as follows.

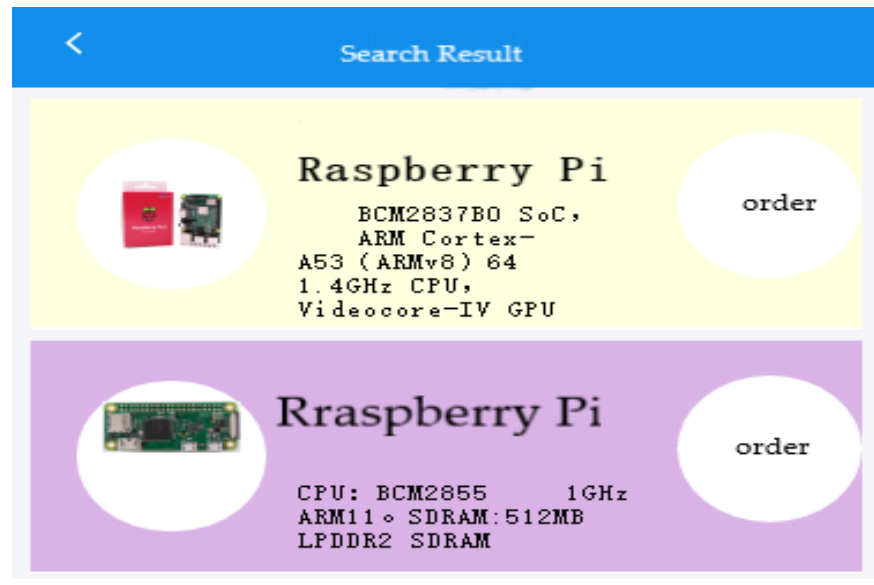

Fig. 3. The search result display page.

\section{My Reservation}

Equipment Reservation Status

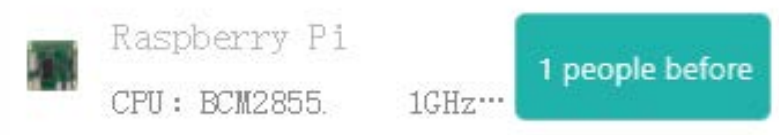

Fig. 4. The reservation display page.

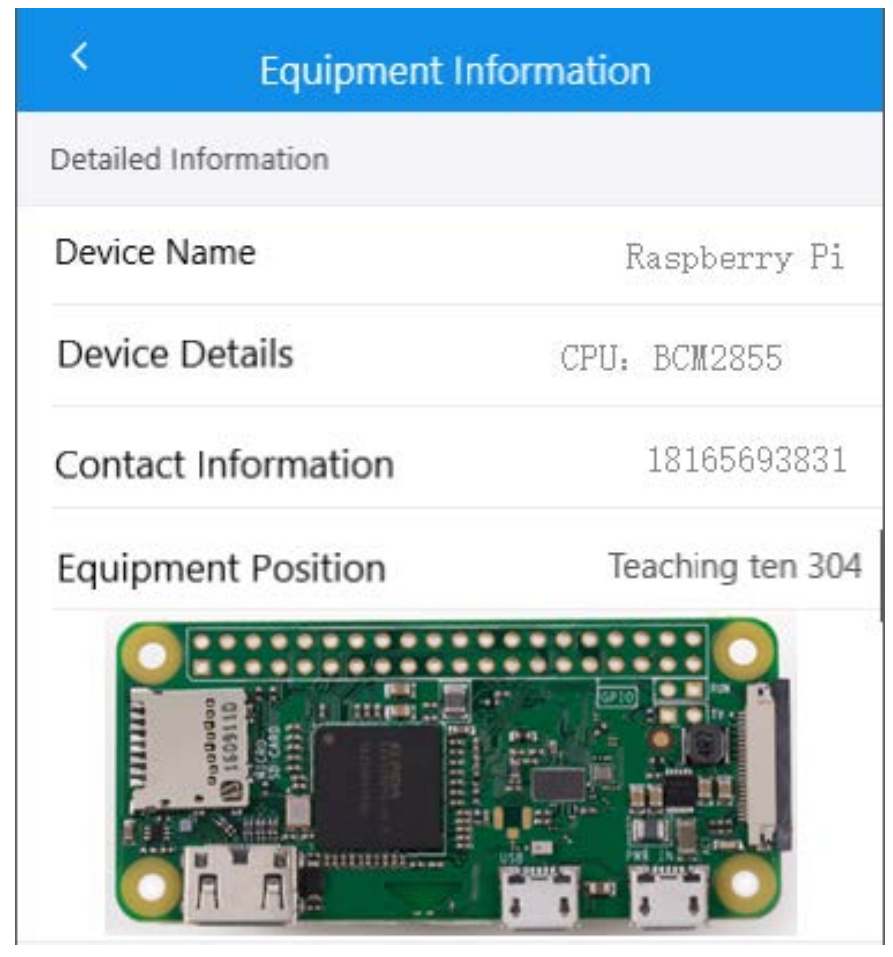

Fig. 5. The equipment information display page.

\section{THE REMOTE CONTROL FUNCTION}

The remote control function of the teaching platform in this paper refers to providing the device usage right reservation and the device remote debugging right transfer management function, establishing a link channel between the user and the device transferor, so that remote program debugging can be realized through the remote control software. This function can be applied to course teaching as well as to innovative talent development activities.

The classroom teaching of professional courses focuses on the theoretical knowledge points required for training in practice. The current classroom teaching is generally carried out in the classroom, and the experimental equipment is not easy to bring into the classroom for demonstration, so the classroom teaching and practical training are separated each other. Using the shared teaching platform proposed in this paper, the teaching content discussed can be visually presented through video and remote control of experimental equipment, which is convenient for students to understand and discuss in class, in the meantime the classroom discussion ideas of students can be verified directly by remote operation.

For the needs outside the curriculum, such as practical skills training in the process of college student innovative and entrepreneurial activities, the shared practical teaching and training platform proposed in this paper can provide the convenience of mutual learning and communication.

College student innovation and entrepreneurship projects, including electronic design competitions, have become an important part of comprehensive innovation ability training. Due to the limitations of the pre-requisite courses, the general 
professional courses are often set in the junior year. While innovative training programs are for the sophomores and juniors, and are oriented to all majors in the school. The teaching platform in this paper enables the composite innovative talents to use the professional laboratory equipment resources to realize remote debugging, and use the shared function to learn and communicate, which can expand the benefit of professional curriculum teaching.

\section{CONCLUSION}

The practical teaching platform based on WeChat secondary development technology designed in this paper has convenient operation, rapid communication, data sharing, remote program debugging, mobile phone network login, experience sharing and discussion, sharing laboratory and course teaching functions. The characteristics of high utilization rate and good interactivity provide a new mode of shared practical teaching for the innovation training.

\section{ACKNOWLEDGMENT}

This work is supported by (13001802) the research project on the reform of higher education in Hebei, China.

\section{REFERENCES}

[1] Shanwei Ge, "The Development and Implementation of patient medical service system Based on WeChat platform,” Nanjing Normal University, 2015.

[2] Yiyu Yang, "The design and implementation of university teaching management service system based on WeChat platform development model,” Information and Computer, pp. 85-86, July 2016.

[3] Yiming Sun, Qinghui Liu, “Students’ Psychological Counseling System based on Cloud-based College,” Information and Computer (Theoretical Edition), pp. 50-51, July 2016.

[4] Ying Xie, "Exploration of Biochemistry Assisted Instruction with Network Teaching Platform," Journal of Jilin Institute of Chemical Technology, pp. 51-53, June 2010.

[5] Xiaojun Wei, "Analysis of the development of online education platform at home and abroad,” Theory Research, pp. 144-145, January 2012.

[6] Yuping Wang, Wei-Chieh Fang, Julia Han, "Exploring the affordances of WeChat for facilitating teaching, social and cognitive presence in semi-synchronous language exchange,” Australasian Journal of Education Technology, 32(4), pp. 18-37, 2016.

[7] Zhang Mei, Shan Yan, Chen Man, "Construction of medical information retrieval network item based on blackboard network teaching platform," $5^{\text {th }}$ International Conference on Computational and Information Sciences, pp. 396-398, 2013.

[8] Zijuan Shi and Gaofeng Luo, "Application of WeChat teaching platform in interactive translation teaching," International Journal of Emerging Technologies in learning, 11(9), pp. 71-75, 2016.

[9] Caiyun Gao and Jingyan Wang, "Mobile teaching of digital mapping based on the wechat official account admin platform," International Journal of Emerging Technologies in learning, 12(7), pp. 56-67, 2017.

[10] Yuegang Fu and Zhiying Liu, "Optical test course teaching practice on WeChat public platform,” 14th Conference on Education and Training in Optics and Photonics, v 10452, 2017. 